\title{
EMOTION ANALYSIS OF TWEETS USING DEEP LEARNING TECHNIQUES
}

\author{
Sahil Bhatnagar, Sagar Malik and Niladri Chatterjee \\ Indian Institute of Technology, Delhi, India
}

\begin{abstract}
Microblogging is one of the most prominent forms of communication among internet users today. These services offer users a tool to share opinions on a plethora of issues and aspects of their lives, and hence, they are rich data sources for textual analysis, and offer vast potential to gain insight into the way people communicate. We examine the task of emotion analysis of twitter data and design a neural network architecture for the task of classifying each tweet into one of five emotion categories: joy, sadness, anger, laughter and fear. We also show a method to automatically collect and tag a corpus of data for emotion analysis. Our experiments showed that our models outperform current state of the art techniques for the proposed problem.
\end{abstract}

\section{KEYWORDS}

Emotion Analysis, Deep Learning, LSTM, Twitter

\section{INTRODUCTION}

Microblogging sites are a source of varied opinions and emotions, with millions of messages constantly exchanged on a vast spectrum of domains. The active user base of Twitter alone stands at over 330 million users (Statista, 2018). People express opinions about their life, the products or services they use, of problems they face and on social, religious and political issues. Many of these opinions have emotions associated with them, and understanding these emotions has vast applications for different organizations. For example, a company might want to know how people respond to their current marketing campaign, social organizations and NGOs may want to understand how people feel about present social issues or political parties may want to understand what people think of their policies and/or candidates. Given the vast number of users on microblogging sites, and especially on Twitter, who express their thoughts and opinions on the above issues, it is an important avenue for a range of organizations to mine and analyze the emotions of the users. Additionally, emotion analysis of text can serve as a step in the pipeline for other complex tasks, such as accessibility frameworks involving text-to-speech, where having knowledge of the emotion of the sentence can lead to more nuanced speech output.

Emotions expressed by humans have been classified into multiple types, from Ekmans list of 6 basic emotions to the more comprehensive Plutchik's Wheel of Emotions (Plutchik, 1989). In our paper, we condense the emotions analyzed into 5 categories: happiness, sadness, anger, laughter and fear (Chin et al., 2016). Thus, our problem is fundamentally a multi-class classification task. The fundamental pipeline for the task is as described in figure 2.

For our task, we restricted the data to the category of political tweets. We collected a corpus of over 18000 tweets evenly distributed among the top 2016 US presidential election candidates: Donald Trump, Hillary Clinton, Bernie Sanders and Ben Carson, which we automatically tagged using methodology described later. For experiments involving Hindi, we use a corpus of around 20000 Hindi tweets evenly distributed amongst various Indian political leaders and parties. We perform some basic linguistic analysis and describe some basic pre-processing and data cleaning techniques that we applied. We then describe and analyze deep learning techniques to model the classification problem, and proceed to create a model which outperforms present state-of-the-art techniques in the domain of emotion analysis of tweets.

We have organized the rest of this paper into 7 following sections. In section 2, we do survey current literature and discuss previous works on sentiment and emotion analysis of tweets. In section 3, we discuss our 
technique to automatically collect and annotate tweets. In section 4, we describe our pre-processing steps and analyze the resulting corpus. In section 5 , we present our baseline neural network model. In section 6 , we present our new state of the art long short term memory (LSTM) neural network model. In section 7, we analyze the results, compare the different models and give insights into the same. We conclude and discuss some avenues of future research in section 8 .

\subsection{Contributions}

The contributions of our paper are as follows:

- We present a scalable, automated method of collecting and annotating tweets with their respective emotion. This allows us to collect arbitrary large corpora of data for emotion analysis tasks

- We devise a model which allows us to solve the problem for tweets of any language

- We perform experimental evaluation of our model prove the efficacy of our solution and show that it performs better than previous work

\section{LITERATURE SURVEY}

Sentiment analysis at different levels of granularity has been worked on and is a significant field of interest for NLP researchers. Recently, there has been a lot of research on sentiment analysis of microblogging data in particular. Some of the popular early work has been done by Go et al. (2009), Bermingham and Smeaton (2010) and Pak and Paroubek (2010). Much of their work has been done to classify tweets into 3 basic categories of sentiments, positive, negative and neutral. The fundamental models used are built using Naive Bayes, MaxEntropy and SVMs. Many researchers have used distant learning methods to curate the datasets, for example, using tweets with emoticons to serve as noisy labels to the data. For the feature space, Go et al. (2009) used a combination of unigrams and bigrams based feature spaces along with features derived from POS tags. Pak and Paroubek (2010) used similar features, but differed in the way they curated their dataset and their use of entropy measures to choose more discriminative n-gram features. Barbosa and Feng (2010) additionally used Twitter specific syntax features like hashtags, links and exclamation tags in addition to using prior polarity of words (derived from various sentiment lexicons) and their POS tags. Agarwal et al. (2011) furthered the ideas of Barbosa and Feng (2010) by using real valued polarity lexicon like WordNet, and gave an in-depth analysis of the effect of various features. Their conclusions stated that tweet syntax features only marginally improved classification accuracy, while the most important features were the prior-polarity of parts-of-speech.

Fundamentally, the above works focus on feature engineering, which is different to our approach of learning the features automatically while training our model on large distantly supervised tweet corpora. Tang et al. (2014) have worked on techniques to automatically learn sentiment specific word embeddings (SSWE) and have developed a corresponding deep learning model "Coooolll". Severyn and Moschitti (2015) performed sentiment analysis using deep convolutional neural networks.

In the explicit domain of emotion classification, Alm et al. (2005) worked on emotion analysis of story text, and created features using WordNet lexicon, POS tags, punctuation and bag of words. Microsoft (2015) has publicly published some of their work on the task of emotion classification using deep learning on annotated data from the Mechanical Turk. Their model was a relatively simple 3-layer deep neural network used along with word embeddings obtained from the public domain such as GloVe (Pennington et al., 2014). Thomas et al. (2014) used a multinomial Naive Bayes approach using unigram features for the identification of emotions in the text to one of seven classes, as defined in the ISEAR (Scherer and Wallbott, 1994) dataset. Razek and Frasson (2017) built individual models for each emotion using a Dominant Meaning tree model, and chooses the emotion with the highest score for a given sentence representation.

\section{AUTOMATED CORPUS COLLECTION}

We used a combination of Twitter API and web-scraping techniques to collect tweets. We only considered tweets which contained keywords such as "Donald Trump", "Trump", "Hillary", "Clinton", "Sanders", "Modi", 
"BJP", "Gandhi", "Congress" etc. in order to restrict them to the primary subjects. The tweets scraped in English were primarily concerned with the 2016 US elections and the candidates, whereas for Hindi, recent tweets in the run up to the 2019 Indian Parliamentary elections about various key figures have been considered.

Multiple studies have been done on the correlation of the emojis used in a tweet and the corresponding emotion of the text. There has been a strong correspondence between the emotion intended and the use of emojis is (Novak et al., 2015). Additionally, since tweets are restricted in length, they generally comprise of only 1 sentence, or a couple of related sentences. Hence, the emojis used can be expected to correspond to the emotion of the tweet at large. Thus, we used a distant supervision model of using the emojis to assign emotion classes to each tweet. We collected millions of tweets of which around 100,000 contained emojis. Of those, around 44000 of them contained emojis which were associated with an emotion in our lexicon. Figure 1 gives the lexicon we used to annotate the tweets with the corresponding emotion. When considering Hindi tweets, the lexicon stayed the same except for the emotions associated with the National Flag emojis.

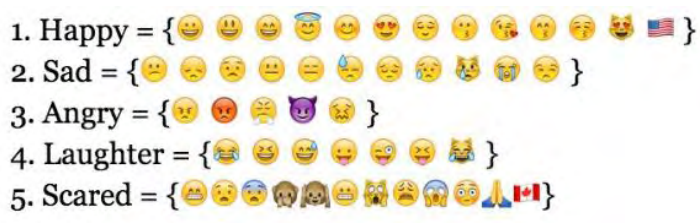

Figure 1. The lexicon used to automatically tag tweets to emotion classes (Chin et al., 2016)

In tweets which contained more than one emoji, we assigned the emotion which was associated with the maximum number of emojis in that tweet. In the cases of a tie, we rejected that tweet.

\section{CORPUS ANALYSIS AND PROCESSING}

The distribution of emotions in the dataset is given in table 1. For the ISEAR data, there are 1076 sentences for each of the 7 emotion classes (Scherer and Wallbott, 1994).

Table 1. Confusion matrix generated on a randomized blind test set of 4728 tweets

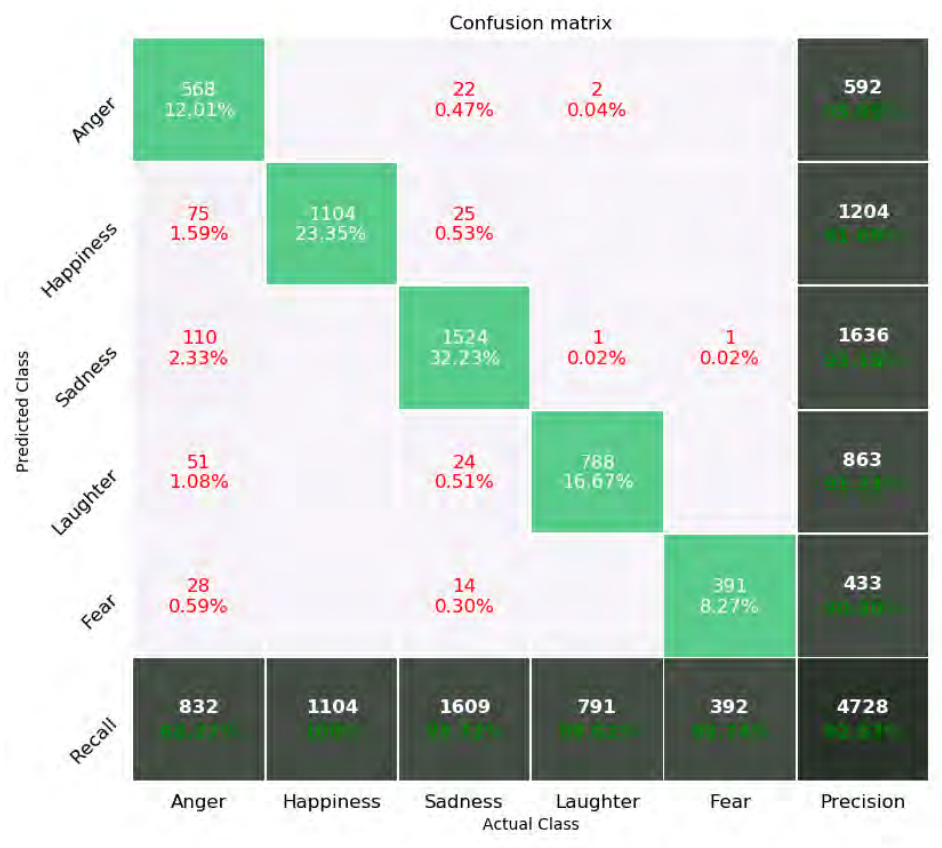


Table 2. Per emotion count of tweets for either dataset

\begin{tabular}{llllll}
\hline Language & Joy & Fear & Sadness & Anger & Laughter \\
\hline English & 2843 & 2439 & 6740 & 12241 & 7316 \\
Hindi & 5164 & 2889 & 3249 & 8043 & 2573 \\
\hline
\end{tabular}

Before passing the tweets in the neural network architecture, some basic preprocessing steps were performed (Garg and Chatterjee, 2014). These are crucial in order to reduce the size of the vocabulary while keeping only relevant words. The following were the steps to clean and process the tweets:

- Tokenization - The most important of these steps is tokenizing the tweet, for which we used the nltk (Bird et al., 2009) TweetTokenizer. This allowed us to get tokens while maintaining the integrity of URLs, @mentions and hashtags, along with punctuation like exclamation marks.

- Stopword Removal - We then removed stopwords which generally do not play a role in determining sentiments, like articles and some prepositions.

- $\quad$ Filtering - We removed URLs, @ mentions and numbers using specially constructed regexes, and also removed punctuation like quotes, commas etc

- Lemmatization - We used the nltk's WordNet lemmatizer to reduce words. This played a significant role by reducing the size of the vocabulary by almost $15 \%$. We couldn't apply this step for Hindi, since reliable lemmatizers aren't readily available for the language.

\section{MODELS}

Instead of working on hand-crafted features to perform emotion analysis we abstracted that task to neural networks in addition to word embeddings which capture semantic meaning of words. In the first sub-section, we describe a basic neural network architecture and associated models with their potential shortcomings. In the second sub-section, we describe the architecture of the LSTM based deep-learning model that outperformed the standard models. For all models, a masking layer was used to pad shorter tweets/sentences with a token word in order to keep all sentences of the same size.

\subsection{Neural Networks}

The first step in the architecture is to represent words in a vector space format. We used pre trained embeddings from the Global Vectors for Word Representation (GloVe) (Pennington et al., 2014) project to represent each word in a 100-dimensional vector. Further, in order to get a vector representation of the sentence constituted by the words i.e. the sentence vector, we tried 2 different approaches.

- Mean of vectors of words in a sentence - Since word-vectors also represent in some way, the sentiment behind every word, taking the mean across all the words describes the overall emotion vector for the sentence (Arora et al., 2016).

- Element-wise max across the word vectors - If we assume that underlying emotions of words are also captured by the word embedding, with different emotions being mapped to different dimensions, then taking the max of the dimensions may give us the emotion vector of the sentence.

Once a sentence vector is obtained, it was passed into a Neural Network with 5 Dense Layers. The models obtained were much faster than other deep learning models with considerably smaller number of learning parameters and had fairly high accuracy. The problem while taking the mean across all the words is that equal importance is given to all the words in a sentence whereas some words can be neutral while others can strongly represent an emotion like anger or laughter. The models does not capture the relationships between the words of a sentence. Therefore, the semantic meaning of the sentence gets lost if a sentence contains both positive and negative words. To overcome the above challenges, we use LSTM models.

\subsection{Long-Short Term Memory Neural Networks}

LSTM networks can be applied to get appropriate semantic meaning of the sentence as it effectively detects the keywords while attenuating less important words in the sentence automatically by switching on and off the gates within the LSTM cells. LSTMs being a special kind of Recurrent Neural Network are able to resolve long term dependencies (Hochreiter and Schmidhuber, 1997). 
For training the network, we use the backpropagation algorithm with an adaptive learning rate (Kingma and $\mathrm{Ba}, 2014)$. The LSTM layer is further stacked with dense layers and finally, to obtain a probability vector we use a softmax classifier given below:

$$
P(y=j \mid x)=\frac{e^{x^{T} w_{j}}}{\sum_{k=1}^{k} e^{x^{T} w_{k}}}
$$

The outline of the neural network architecture is illustrated in the figure given below.

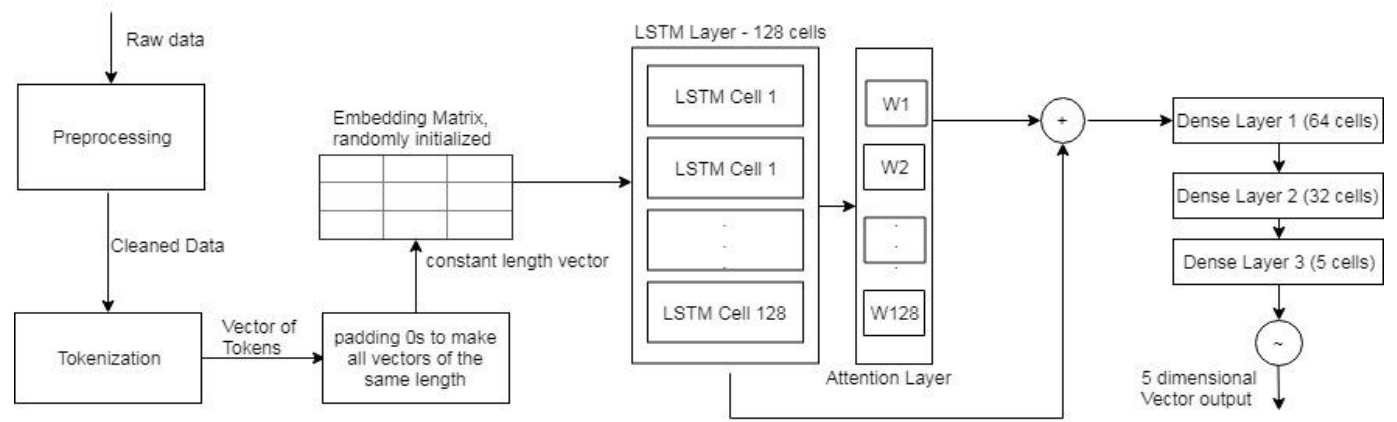

Figure 2. Neural Network architecture corresponding to the LSTM based model

To obtain word embeddings required for the LSTM models we used the following 2 different approaches.

- Using pre-trained Embeddings: We used the GloVe Embedding which is already pre-trained on twitter data (Pennington et al., 2014), thus we had vector representation of nearly all the words of our collected English dataset. Therefore, our model could not only have a representation for all words in our training set, but also handle most new words in the test or validation set.

- Training an Embedding Layer: To make our approach more generic we used an embedding layer instead of using pre-trained embeddings. We uniquely indexed the words in the training data and then passed the resultant sentence vector into the embedding layer on which further layers of the models were stacked. The embedding matrix gets trained along the network. We observed the variation in the model's performance with the number of unique words in our training data. Since our embedding matrix was trained on limited number of words, on encountering a new word in test set, we assign a default index of the embedding matrix to these words. Given that the model is learning word representation while training, it is able to learn local context of the words in the data instead of relying on the universal meaning. This idea is extremely powerful, since it allows us to use the same technique to work with different languages and contexts, hence allowing us to abstract away the differences in language structures and semantics.

Finally, we added an attention layer stacked immediately after the LSTM. An attention mechanism allows the model to gain a finer understanding of the different parts of the sentence/tweet, potentially allowing for clearer identification of emotions in the different parts.

\section{BASELINE ACCURACY}

In order to understand how well our model performs, we determine the following benchmarks

- Probabilistic baseline - Since the task is a 5-class classification, random probability to get a correct result is $20 \%$.

- Skewed probabilistic baseline - In light of the fact that the data is skewed and the dominant class accounts for almost $40 \%$ of the data, a naive classifier giving the dominant class as the only answer would result in about $40 \%$ accuracy.

- Past research - We will evaluate and compare the results of contemporary models such as the one proposed by Microsoft (2015) on our dataset, as well as evaluate our models (with minimal modifications) on the standardized ISEAR (Scherer and Wallbott, 1994) dataset and compare the results with those achieved by Thomas et al. (2014) and Razek and Frasson (2017). For comparing results on the Hindi data, we evaluated the model proposed by Microsoft (2015) on our data and compared the results. 


\section{EXPERIMENTS}

We performed two basic experiments with our neural network architectures. The first was a 5-class emotion analysis as described in the introduction, where we classify the dominant emotion of a tweet into one of the following five emotions- joy, sadness, anger, laughter and fear. We split the tweets according to language and into 3 sets; train, verify and test. The training and test sets were made by using a 80-20\% split of the dataset for each language. Further, $20 \%$ of the total training set was used as a verification set to train and improve the model, and comprised of a total of 17534 tweets for the Hindi set, and 26842 tweets for the English set. The remainder of tweets were used to test the developed models, and results were measured using the accuracy measure, as well as per-class precision and recall via a confusion table. The division of the tweets was done randomly after shuffling the dataset. The ADAM optimization algorithm was used for training.

The second experiment was performed using the ISEAR dataset in order to compare the performance of fundamental neural network architecture. Thus, using the same architecture as proposed, we trained a new model based on the exact same architecture with the minor modification of having a 7-node final layer instead of a 5-node layer in order to perform a 7-class emotion analysis. In order to make a direct comparison to the models proposed by Thomas et al. (2014) and Razek and Frasson (2017), we used the same train-test split of the dataset as they had in their experiments.

\section{RESULTS}

The first trend noticed was that accuracy of models increased with the number of unique words considered (Table 3). This could be explained as some words that may not be frequent in the dataset may change the overall sentiment of the sentence and to the fact that the models couldn't capture the sentiment of new unseen words in the test data set. The variation was less when a pre-trained embedding was used instead of training an Embedding layer. The best neural network model had an accuracy of $86.8 \%$. Such high accuracy could be attributed to the embedded sentiment data in the pre-trained GloVe embeddings (Pennington et al., 2014). Hence, the computed sentence vector must also be carrying some of this meaning.

Table 3. Accuracy obtained using the basic Neural Network

\begin{tabular}{ll}
\hline $\begin{array}{l}\text { Neural Network Model } \\
\text { (Sec 5.1) }\end{array}$ & Accuracy \\
\hline Mean Model (1000 words) & $82.07 \%$ \\
Mean Model (2000 words) & $82.5 \%$ \\
Mean Model (3000 words) & $83.2 \%$ \\
Mean Model (all words) & $\mathbf{8 6 . 8 \%}$ \\
Max Model & $84.9 \%$ \\
\hline
\end{tabular}

Table 4. Comparison of accuracy: English Dataset

\begin{tabular}{ll}
\hline Model & Accuracy \\
\hline Microsoft (2015) & $89.47 \%$ \\
Neural Network & $86.8 \%$ \\
LSTM Model & $90.06 \%$ \\
LSTM Model w/ Attention & $\mathbf{9 2 . 4 8 \%}$ \\
\hline
\end{tabular}

Table 5. Comparison of accuracy: Hindi Dataset

\begin{tabular}{ll}
\hline Model & Accuracy \\
\hline LSTM & $86.36 \%$ \\
LSTM w/ attention & $\mathbf{8 8 . 5 0 \%}$ \\
\hline
\end{tabular}

The LSTM models outperformed above models because these models also learnt the semantic relationship between the words in a sentence. Implicit relationships between words and their corresponding parts of speech as well as their usage in specific context and their spatial relationship with other words are captured by the 
LSTM model, which are otherwise lost using the simple Neural Network strategy (Hochreiter and Schmidhuber, 1997). As expected from previous research, the implicit incorporation of the above features led to better accuracy using the LSTM model. The accuracy of the models may further be increased by increasing the size of the dataset and thus reducing the probability of discovering new words. The model trained in less than 5 minutes on an Nvidia Tegra K80 gpu, thus it is relatively lightweight in terms of resources required to train.

Table 6. Per emotion count of tweets for either dataset

\begin{tabular}{llllll}
\hline Model & Joy & Fear & Sadness & Anger & Laughter \\
\hline Microsoft (2015) & 0.92 & 0.91 & 0.90 & 0.77 & 0.93 \\
LSTM w/ attention & $\mathbf{0 . 9 6}$ & $\mathbf{0 . 9 4}$ & $\mathbf{0 . 9 4}$ & $\mathbf{0 . 8 0}$ & $\mathbf{0 . 9 5}$ \\
\hline
\end{tabular}

We also plot the confusion matrix, from which we see that the model possesses very high precision and recall for almost all classes. As compared to the study conducted by Microsoft (2015), our model outperforms them consistently across all emotions by at least 3 percentage points in the f-score measure. The LSTM model inherently develops a better understanding of sentence context as compared to other simple neural network or bag of words models, which is why we believe we see such higher per-class precision and recall.

The second experiment on the ISEAR (Scherer and Wallbott, 1994) dataset, finds our results convincingly better than those obtained by Thomas et al. (2014) and Razek and Frasson (2017). This shows the general ability of our model to outperform manual feature extraction based methods of emotion analysis.

Table 7. Comparison of per-class F-score: ISEAR Dataset

\begin{tabular}{llllllll}
\hline Model & Joy & Fear & Sadness & Anger & Guilt & Disgust & Shame \\
\hline Razek and Frasson (2017) & 0.58 & 0.56 & 0.67 & 0.66 & 0.50 & 0.47 & 0.55 \\
LSTM w/ attention & $\mathbf{0 . 9 6}$ & $\mathbf{0 . 8 1}$ & $\mathbf{0 . 9 0}$ & $\mathbf{0 . 8 0}$ & $\mathbf{0 . 7 8}$ & $\mathbf{0 . 8 9}$ & $\mathbf{0 . 7 8}$ \\
\hline
\end{tabular}

Table 8. Comparison of per-class Accuracy: ISEAR Dataset

\begin{tabular}{llllllll}
\hline Model & Joy & Fear & Sadness & Anger & Guilt & Disgust & Shame \\
\hline Thomas et al. (2014) & $78.3 \%$ & $83.06 \%$ & $\mathbf{7 9 . 1 6 \%}$ & $68.94 \%$ & $80.67 \%$ & $69.76 \%$ & $78.84 \%$ \\
LSTM w/ attention & $\mathbf{9 2 . 8 2 \%}$ & $\mathbf{8 7 . 0 8 \%}$ & $70.0 \%$ & $\mathbf{7 3 . 8 7 \%}$ & $\mathbf{9 0 . 7 1 \%}$ & $\mathbf{8 1 . 0 1 \%}$ & $\mathbf{8 0 . 3 6 \%}$ \\
\hline
\end{tabular}

Finally, we analyze our LSTM based models on the Hindi dataset we collected. The relatively high accuracy obtained verifies our claim that our proposed architecture can also work with tweets in a different language too. Note that models depending on pre-trained embeddings would not easily work with such a task. Similarly, hand-crafted features would potentially be different and hence, not scalable to train on multiple languages.

\section{CONCLUSION}

In our paper, we have successfully designed a strategy to automatically collect twitter corpus and tag it using distant learning techniques, thus allowing us to continuously extract any amount of data to train and test our models. Further, we used it to collect corpus to train different deep learning techniques to perform emotion analysis on tweets, with the LSTM approach, as expected, giving the best results. Additionally, we can see that the results obtained beat current state-of-the-art techniques.

For future work, we plan on augmenting our embedding layers, using both pre-trained embeddings like GloVe and using them to initialize our layer, as well as incorporating different linguistic features like POS tags to better train the embedding. Finally, we think working on mixed language tweets would prove an interesting challenge. 


\section{REFERENCES}

Apoorv Agarwal, Boyi Xie, Ilia Vovsha, Owen Ram-bow, and Rebecca Passonneau. 2011. Sentiment analysis of twitter data. In LSM '11 Proceedings of the Workshop on Languages in Social Media, pages 30-38, Portland, Oregon.

Cecilia Ovesdotter Alm, Dan Roth, and RichardSproat. 2005. Twitter sentiment analysis with deep convolutional neural networks. In HLT '05 Proceedings of the conference on Human Language Technology and Empirical Methods in Natural Language Processing, pages 579-586, Vancouver, British Columbia, Canada.

Sanjeev Arora, Yingyu Liang, and Tengyu Ma. 2016. A simple but tough-to-beat baseline for sentence embeddings.

Luciano Barbosa and Junlan Feng. 2010. Robust sentiment detection on twitter from biased and noisy data. In Proceedings of the 23rd international conference on computational linguistics: posters, pages36-44. Association for Computational Linguistics.

Adam Bermingham and Alan F Smeaton. 2010. Classifying sentiment in microblogs: is brevity an advantage? In Proceedings of the 19th ACM international conference on Information and knowledge management, pages 1833-1836. ACM

Steven Bird, Ewan Klein, and Edward Loper, 2009. Natural Language Processing with Python. O'Reilly Media.

Delenn Chin, Anna Zappone, and Jessica Zhao. 2016. Analyzing twitter sentiment of the 2016 presidential candidates. American Journal Of Science and Research.

Yogesh Garg and Niladri Chatterjee. 2014. Sentiment analysis of twitter feeds. In International Conference on Big Data Analytics, pages 33-52. Springer.

Alec Go, Richa Bhayani, and Lei Huang. 2009. Twitter sentiment classification using distant supervision. CS224N Project Report, Stanford, 1(12).

Sepp Hochreiter and J ürgen Schmidhuber. 1997. Long short term memory. Neural computation, 9(8):1735-1780.

Diederik P Kingma and Jimmy Ba. 2014. Adam: Amethod for stochastic optimization. arXiv preprint arXiv:1412.6980.

Microsoft. 2015. Emotion detection and recognition from text using deep learning. www.microsoft.com/developerblog.

Petra Kralj Novak, Jasmina Smailovi'c, Borut Sluban, and Igor Mozetǐc. 2015. Sentiment of emojis. PloS one, 10(12):e0144296.

Alexander Pak and Patrick Paroubek. 2010. Twitter as a corpus for sentiment analysis and opinion mining. In $L R E c$, volume 10

Jeffrey Pennington, Richard Socher, and Christopher Manning. 2014. Glove: Global vectors for word representation. In Proceedings of the 2014 conference on empirical methods in natural language processing (EMNLP), pages 1532-1543.

R. Plutchik. 1989. Emotion: Theory, Research, and Experience. v. 4. Acad. Press.

Mohammed Abdel Razek and Claude Frasson. 2017. Text-based intelligent learning emotion system. Journal of Intelligent Learning Systems and Applications, 9(01):17.

Klaus R Scherer and Harald G Wallbott. 1994. Evidence for universality and cultural variation of differential emotion response patterning. Journal of personality and social psychology, 66(2):310.

Aliaksei Severyn and Alessandro Moschitti. 2015. Emotions from text: machine learning for text-based emotion prediction. In Proceedings of the 38th International ACM SIGIR Conference on Research and Development in Information Retrieval, pages 959-962, Santiago, Chile.

Statista. 2018. Twitter: Number of active users. www.statista.com/statistics/282087/number-of-monthlyactive-twitterusers.

Duyu Tang, Furu Wei, Bing Qin, Ting Liu, and Ming Zhou. 2014. Coooolll: A deep learning system for twitter sentiment classification. In Proceedings of the 8th International Workshop on Semantic Evaluation, pages 208-212, Dublin, Ireland.

Bincy Thomas, P Vinod, and KA Dhanya. 2014. Multiclass emotion extraction from sentences. International Journal of Scientific \& Engineering Research, 5(2). 